\title{
LINEAR STOCHASTIC DIFFERENTIAL EQUATIONS WITH FUNCTIONAL BOUNDARY CONDITIONS
}

\author{
By Aureli Alabert ${ }^{1}$ And Marco Ferrante ${ }^{2}$ \\ Universitat Autònoma de Barcelona and Università degli Studi di Padova
}

\begin{abstract}
We consider linear $n$th order stochastic differential equations on $[0,1]$, with linear boundary conditions supported by a finite subset of $[0,1]$. We study some features of the solution to these problems, and especially its conditional independence properties of Markovian type.
\end{abstract}

1. Introduction. It is well known that, under suitable Lipschitz and growth conditions on the coefficients, a classical Itô stochastic differential equation

$$
X(t)=\xi+\int_{0}^{t} b(s, X(s)) d s+\int_{0}^{t} \sigma(s, X(s)) d W(s),
$$

where $W$ is a Wiener process and $\xi$ is a $\mathcal{F}_{0}$-measurable random variable for a given nonanticipating filtration $\left\{\mathcal{F}_{t}, t \geq 0\right\}$ of $W$, has a unique strong solution which is a Markov process.

If $\xi$ is not $\mathcal{F}_{0}$-measurable or the coefficients $b, \sigma$ are random and nonadapted, then any reasonable interpretation of $X$ in (1.1) will not be an $\mathcal{F}_{t}$-adapted process and, unless $\sigma$ is a constant, we need to use some anticipating stochastic integral to give a sense to the equation. In these cases, the solution is not a Markov process in general.

Still another setting that leads to anticipation is the case of boundary conditions. That means, the first variable of the solution process is no longer a datum of the problem, time runs in a bounded interval, say from 0 to 1 , and we impose a relation $h(X(0), X(1))=0$ between the first and the last variables of the solution. In this situation, the fact that the solution will not be Markovian is quite intuitive, since the strong relationship between $X(0)$ and $X(1)$ will prevent the independence of $X(0)$ and $X(1)$ from holding, even when conditioning to $X(a), a \in] 0,1$, except maybe in some very particular cases.

On the other hand, it may also seem intuitive that the following weaker conditional independence property can hold true: For any $0 \leq a<b \leq 1$, the $\sigma$-fields $\sigma\{X(t), \quad t \in[a, b]\}$ and $\sigma\{X(t), \quad t \in] a, b\left[{ }^{c}\right\}$ are conditionally independent given $\sigma\{X(a), X(b)\}$. We will denote it by

$$
\sigma\{X(t), t \in[a, b]\} \underset{\sigma\{X(a), X(b)\}}{\|} \sigma\{X(t), t \in] a, b\left[^{c}\right\} .
$$

Received March 2002; revised October 2002.

${ }^{1}$ Supported by CIRIT Grant SGR99-87 and DGESIC Grant BFM2000-0009.

${ }^{2}$ Supported by MURST Grant COFIN9901244421.

AMS 2000 subject classifications. $60 \mathrm{H} 10,60 \mathrm{~J} 25$.

Key words and phrases. Linear stochastic differential equations, conditional independence, Markov property, Markov fields, convergence of conditional expectations. 
Now $X(0)$ and $X(1)$ are on the same side in relation (1.2), so that the boundary condition does not seem to cause the problem seen above. But the following example shows that this is wrong:

EXAMPle 1.1. Consider the problem

$$
\begin{aligned}
& \dot{X}(t)=f(X(t)) d t+\dot{W}(t), \quad t \in[0,1], \\
& h(X(0), X(1))=0,
\end{aligned}
$$

where the noise appears additively, and assume that a unique solution exists and that the boundary condition given by $h$ does not reduce to an initial or final condition. Then, relation (1.2) holds if and only if $f(x)=\alpha x+\beta$, for some constants $\alpha$ and $\beta$. This was proved by Nualart and Pardoux [16].

The processes satisfying (1.2) were called reciprocal processes by Bernstein [5]. The concept arose directly from Schrödinger ideas on the formulation of quantum mechanics. More recent research on such processes has been carried out by Jamison [12], Krener [14], Krener, Frezza and Levy [15], Thieullen [20] and Zambrini [21].

Other names can be found in the literature to refer to the same concept. A reciprocal process is a one-parameter Markov field in Paul Lévy's terminology, and is also called a quasi-Markov process, a local Markov process and a Bernstein process. We shall simply call them Markov fields (see Definition 4.1).

EXAMPLE 1.2. Now consider the problem

$$
\begin{aligned}
& \ddot{X}(t)+f(X(t), \dot{X}(t))=\dot{W}(t), \quad t \in[0,1], \\
& X(0)=c_{1}, \quad X(1)=c_{2} .
\end{aligned}
$$

This is a second-order stochastic differential equation, and it is natural to ask for conditional independence properties of the two-dimensional process $Y(t)=$ $(\dot{X}(t), X(t))$, since $X(t)$ has $C^{1}$ paths, and therefore it is meaningless to look for this kind of properties for $X(t)$ itself.

Nualart and Pardoux [17] proved that if $Y(t)$ is a Markov field, then, as in Example 1.1, $f$ must be an affine function. Moreover, if $f$ is affine, then $Y$ is not only a Markov field, but a Markov process.

Let us look at this example more closely: note that a boundary condition for a second-order equation has the general form

$$
h(Y(0), Y(1))=(0,0),
$$

where $Y(0)=(\dot{X}(0), X(0))$ and $Y(1)=(\dot{X}(1), X(1))$. However, in Example 1.2 the two scalar conditions do not mix values at 0 and values at 1 of $Y$. The same 
happens, for instance, with the Neumann-type conditions $\dot{X}(0)=c_{1}, \dot{X}(1)=c_{2}$, and the result is the same ( $Y$ Markov field $\Rightarrow f$ affine $\Rightarrow Y$ Markov process).

From these examples and other equations of first and second order that have been studied so far (see, e.g., $[2-4,18]$ ), we learn that:

1. The Markovian properties can be expected only in "linear" cases.

2. The specific Markovian property depends on the actual form of the boundary condition.

It should also be noted that the requirement of linearity on the drift coefficient $f$ is related to the fact that the noise appears additively. Should not this be the case, the Markovian property would occur under a different condition which relates the drift and the diffusion coefficients (see [2] and [3]).

In the present paper we will consider linear stochastic differential equations of arbitrary order with additive white noise. Our boundary conditions will not be restricted to involve the solution process at the endpoints of the time interval, but we will allow them to involve the values at finitely many points inside the interval. They are usually called functional or lateral boundary conditions. Our main goal is to seek which kind of conditional independence properties can be established for the solution. A preliminary work in this direction was published in [1]. Here we considerably refine and extend the results therein. This type of equations was already considered by Russek [19], who proved that the solutions are Markov processes if and only if the lateral conditions fix to a constant the variables $X(t)$, for all points $t$ in the support of the conditions. His techniques, based in the notion of reproducing kernel space, are different from ours.

Our main result (Theorem 4.5) can be stated in the following way: Fix two points $0 \leq a<b \leq 1$ and set $Y(t)=\left(D^{n-1} X(t), \ldots, D X(t), X(t)\right)$, where $n$ is the order of the equation, $X(t)$ is its solution process and $D$ is the time derivative; the process $Y=\{Y(t), t \in[0,1]\}$ satisfies the relation (1.2) if and only if there are no lateral conditions involving points inside and outside the interval $[a, b]$. (Note that this is different from saying that $Y$ is a Markov field.) We also state a conditional independence property for the case when there are conditions that do involve points inside and outside $[a, b]$ (Theorem 4.10). Finally we obtain a result from which Russek's theorem can be trivially recovered (Theorem 4.11). The paper is organised as follows:

In Section 2 we define the statement of the problem and develop some notation and properties that will be needed later.

Section 3 contains the main probabilistic tools: Lemma 3.4 and Proposition 3.7. The first is a characterization of the conditional independence of two random vectors given a function of them. It is the most important ingredient in the proof of Theorem 4.5, but it cannot be applied for certain singular values of $a$ and $b$. For these values, we employ an approximation by the solution of perturbed equations. The approximation argument involves the convergence in $L^{2}$ of a sequence of 
conditional expectations with varying conditioning $\sigma$-fields. Proposition 3.7 gives a sufficient condition for this convergence in a general setting.

In Section 4 we establish the main results. The proofs of Theorems 4.10 and 4.11 will be only sketched, since the procedure is similar to that of Theorem 4.5, with slight modifications.

2. Linear SDE with functional boundary conditions. The present section will be devoted to the statement of the problem, the definition of a solution, and to absolute continuity and approximation results for the solution of an $n$th order linear stochastic differential equation with linear functional boundary conditions.

2.1. Statement of the problem and definition of a solution. Consider the differential operator

$$
L:=D^{n}+a_{n-1} D^{n-1}+\cdots+a_{1} D+a_{0}, \quad D:=\frac{d}{d t},
$$

where $a_{i}$ are continuous functions on $[0,1]$. Let $\{W(t), t \in[0,1]\}$ be a standard Wiener process. We assume that $W$ is the coordinate process in the classical Wiener space $(\Omega, \mathcal{F}, P)$, that means, $\Omega=C_{0}([0,1] ; \mathbb{R})$ is the space of continuous functions on $[0,1]$ vanishing at zero, $\mathcal{F}$ its Borel $\sigma$-field, and $P$ the Wiener measure. We shall deal with the SDE

$$
L[X]=\dot{W}
$$

on $[0,1]$, together with the additional conditions

$$
\sum_{j=1}^{m} \alpha_{i j} X\left(t_{j}\right)=c_{i}, \quad 1 \leq i \leq n,
$$

where $m \geq n, 0 \leq t_{1}<\cdots<t_{m} \leq 1$ are some given points in $[0,1]$ and $\alpha_{i j}, c_{i}$ are real numbers. The matrix of coefficients $\left(\alpha_{i j}\right)$ is assumed to have full rank.

As in the case of ordinary differential equations, (2.1) and (2.2) can be regarded as a first-order system

$$
D Y(t)+A(t) Y(t)=\dot{B}(t), \quad t \in[0,1],
$$

with constraints

$$
\sum_{j=1}^{m} \alpha_{i j} Y_{n}\left(t_{j}\right)=c_{i}, \quad 1 \leq i \leq n,
$$

where $Y(t)=\left(Y_{1}(t), \ldots, Y_{n}(t)\right), Y_{i}(t)=D^{n-i} X(t)$ for $1 \leq i \leq n, B(t)=$ $(W(t), 0, \ldots, 0)$ and

$$
A(t)=\left[\begin{array}{ccccc}
a_{n-1}(t) & a_{n-2}(t) & \cdots & a_{1}(t) & a_{0}(t) \\
-1 & 0 & \cdots & 0 & 0 \\
0 & -1 & \cdots & 0 & 0 \\
\vdots & \vdots & \ddots & \vdots & \vdots \\
0 & 0 & \cdots & -1 & 0
\end{array}\right] .
$$


The lateral condition (2.4) is a special case of the general linear condition

$$
\Lambda[Y]=c,
$$

for $\Lambda$ in the set $\mathcal{L}\left(C\left([0,1] ; \mathbb{R}^{n}\right) ; \mathbb{R}^{n} t\right)$ of linear continuous $\mathbb{R}^{n}$-valued functionals on $C\left([0,1] ; \mathbb{R}^{n}\right)$, and $c \in \mathbb{R}^{n}$. By the Riesz representation theorem, (2.6) can be written as

$$
\int_{0}^{1} d F(t) Y(t)=c
$$

where $F$ is an $(n \times n)$-matrix whose components are functions of bounded variation.

When the right-hand side of (2.3) is a continuous vector function $g$, it is well known (see, e.g., [7] or [11]) that the system

$$
\begin{aligned}
& D Y(t)+A(t) Y(t)=g(t), \quad t \in[0,1], \\
& \int_{0}^{1} d F(t) Y(t)=c,
\end{aligned}
$$

admits a unique solution, which belongs to $C^{1}\left([0,1] ; \mathbb{R}^{n}\right)$, if and only if for some $s \in[0,1]$ (equivalently, for every $s \in[0,1]$ )

$$
\operatorname{det} \int_{0}^{1} d F(t) \Phi^{s}(t) \neq 0
$$

where $\Phi^{s}(t)$ denotes the fundamental matrix solution of $D Y(t)+A(t) Y(t)=0$, that is, $\forall s \in[0,1]$,

$$
\begin{aligned}
& \frac{d}{d t} \Phi^{s}(t)+A(t) \Phi^{s}(t)=0, \quad t \in[0,1], \\
& \Phi^{s}(s)=\mathrm{I},
\end{aligned}
$$

with I the identity matrix. In turn, this is equivalent to say that the homogeneous problem ( $g \equiv 0, c \equiv 0$ ) has only the trivial solution. When hypothesis (H0) holds, the solution to (2.8) is given by

$$
Y(t)=J(t)^{-1} c+\int_{0}^{1} G(t, s) g(s) d s,
$$

where

$$
J(t)=\int_{0}^{1} d F(u) \Phi^{t}(u)
$$

and $G(t, s)$ is the (matrix-valued) Green function associated to $A$ and $F$. An explicit expression for this function is the following:

$$
G(t, s)=J(t)^{-1}\left[\int_{0}^{s} d F(u) J(u)^{-1}-\mathbf{1}_{\{t \leq s\}} \mathrm{I}\right] J(s) .
$$


Under (H0), we define the solution to (2.3) and (2.4) as the $n$-dimensional stochastic process

$$
Y(t)=J(t)^{-1} c+\int_{0}^{1} G(t, s) d B(s),
$$

following the lines of Russek [19], and the solution to (2.1) and (2.2) as the process $\left\{X(t)=Y_{n}(t), t \in[0,1]\right\}$. The Green function (2.10) has bounded variation, so that the Wiener integrals in (2.11) can be interpreted pathwise by means of an integration by parts

$$
\left[\int_{0}^{1} G(t, s) d B(s)\right](\omega)=-\int_{0}^{1} G(t, d s) B(s)(\omega)
$$

[we take into account here that $G(t, 1)=0, \forall t$ ], and therefore $Y$ can be defined everywhere. We shall assume throughout the paper that the solution is interpreted in this pathwise sense. Furthermore, it is not difficult to verify that the process $Y(t)$ so defined is continuous (hence $X(t)$ is a $C^{n-1}$ process) and that, for each $t \in[0,1]$, the mapping $\omega \mapsto Y(\omega)$ from $\Omega$ into $C\left([0,1] ; \mathbb{R}^{n}\right)$ is continuous with the usual topologies.

Notice that, with the notation introduced in (2.7), the particular lateral condition (2.4) corresponds to

$$
d F=\left[\begin{array}{cccc}
0 & \cdots & 0 & \sum_{j=1}^{m} \alpha_{1 j} \delta_{t_{j}} \\
0 & \cdots & 0 & \sum_{j=1}^{m} \alpha_{2 j} \delta_{t_{j}} \\
\vdots & & \vdots & \vdots \\
0 & \cdots & 0 & \sum_{j=1}^{m} \alpha_{n j} \delta_{t_{j}}
\end{array}\right],
$$

where $\delta_{t}$ denotes the Dirac measure at $t$, and that $J_{i k}(t)=\sum_{j=1}^{m} \alpha_{i j} \Phi_{n k}^{t}\left(t_{j}\right)$. Notice also that only the first column of $G(t, s)$ is relevant in (2.11).

Another natural definition of solution for the system (2.3) and (2.4) arises if, for each $\omega$ fixed, we consider the object $\dot{B}(\omega)$ as the derivative of a continuous $\mathbb{R}^{n}$-valued function defined on $[0,1]$, and therefore we regard (2.3) as an equation between distributions. The vector function $Y=\left(Y_{1}, \ldots, Y_{n}\right)$ will be a solution in the distributional sense if for any smooth vector $\varphi=\left(\varphi_{1}, \ldots, \varphi_{n}\right)$ vanishing in the complement of $] 0,1\left[\right.$, with $\int_{0}^{1} \varphi=0$,

$$
\int_{0}^{1}\left(Y(t)+\int_{0}^{t} A(a) Y(s) d s-B(t)\right) \cdot \varphi(t) d t=0
$$

and (2.4) is satisfied. But (2.13) amounts to say that there exists a constant $Y(0)$ such that

$$
Y(t)-Y(0)+\int_{0}^{t} A(s) Y(s) d s=B(t), \quad t \in[0,1],
$$

and a fortiori we find that $Y$ must be a continuous function. It is easily seen that both concepts of solution coincide. 
It is also possible to give a meaning to system (2.1) and (2.2) with a right-hand side in (2.1) of the form $\sigma\left(X(t), D X(t), \ldots, D^{n-1} X(t)\right) \dot{W}(t)$, through the use of anticipating stochastic integrals. The distributional definition of solution does not have a sense in that case. We do not consider this situation here; see however the comments following Definition 4.1.

2.2. On the law of the solution. In the present section we shall prove an absolute continuity result for the law of the solution process $\{Y(t), t \in[0,1]\}$. Here we allow the boundary condition (2.4) to depend on all coordinates of $Y$, since we will use this generality later on.

Let $Y=\left(Y_{1}, \ldots, Y_{n}\right): \Omega \rightarrow C\left([0,1] ; \mathbb{R}^{n}\right)$ be the solution to the problem

$$
\begin{aligned}
& D Y(t)+A(t) Y(t)=\dot{B}(t), \quad t \in[0,1], \\
& \Lambda[Y]=c
\end{aligned}
$$

where $\Lambda$ is any linear operator on $C\left([0,1] ; \mathbb{R}^{n}\right)$ with finite support $\operatorname{supp} \Lambda=$ $\left\{t_{1}, \ldots, t_{m}\right\}$. [We are not assuming here that $\Lambda$ involves only the coordinate function $Y_{n}$, but we do assume that problem (2.14) is well posed.]

If $\left\{s_{1}, \ldots, s_{k}\right\} \subset[0,1]$ is a set containing $\operatorname{supp} \Lambda$, then $\Lambda$ can be regarded as a linear operator on the space of functions $\left(\left\{s_{1}, \ldots, s_{k}\right\} \rightarrow \mathbb{R}^{n}\right) \cong \mathbb{R}^{n \times k}$. We keep the same symbol $\Lambda$ for both interpretations. Denote by $M$ the linear manifold in $\mathbb{R}^{n \times k}$ :

$$
M:=\left\{x=\left(\left(\begin{array}{c}
x_{11} \\
\vdots \\
x_{1 n}
\end{array}\right), \ldots,\left(\begin{array}{c}
x_{k 1} \\
\vdots \\
x_{k n}
\end{array}\right)\right) \in \mathbb{R}^{n \times k}: \Lambda[x]=c\right\} .
$$

PROPOSITION 2.1. With the notation above, the random vector $\left(Y\left(s_{1}\right), \ldots\right.$, $\left.Y\left(s_{k}\right)\right): \Omega \rightarrow \mathbb{R}^{n \times k}$ is absolutely continuous with respect to the Hausdorff measure in $M$.

PROOF. Taking into account that the vector $\left(Y\left(s_{1}\right), \ldots, Y\left(s_{k}\right)\right)$ is Gaussian, it suffices to prove that any open ball in $M$ has a positive probability under the law of this vector.

Fix $x \in M$. Let us see first that there exists $\omega \in \Omega$ such that the function $Y(\omega):[0,1] \rightarrow \mathbb{R}^{n}$ satisfies $\left(Y(\omega)\left(s_{1}\right), \ldots, Y(\omega)\left(s_{k}\right)\right)=x$. Indeed, by simple interpolation, there obviously exists a $C^{\infty}$ function $y:[0,1] \rightarrow \mathbb{R}^{n}$ such that $y_{i} \equiv y_{i+1}^{\prime}, i=1, \ldots, n-1$, and $\left(y\left(s_{1}\right), \ldots, y\left(s_{k}\right)\right)=x$ (therefore $\left.\Lambda[y]=c\right)$. Defining

$$
\omega(t)=y^{1}(t)-y^{1}(0)+\int_{0}^{t}\left(a_{n-1}(s) y^{1}(s)+\cdots+a_{0}(s) y^{n}(s)\right) d s,
$$

we find that $y$ is the solution path $Y(\omega)$ of (2.14). Any open ball $U(x)$ of $M$ centred at a point $x \in M$ has therefore a nonempty inverse image $Y^{-1}(B(x)) \subset \Omega$. 
Moreover, since the mapping $\omega \mapsto Y(\omega)$ is continuous, $Y^{-1}(U(x))$ is open. We get that $P\{Y \in U(x)\}>0$.

REMARK 2.2. Proposition 2.1 remains valid, with a similar proof, if the domain where the problem is considered consists of two disjoint intervals, say $[0, a]$ and $[b, 1]$, instead of a single one (in that case $2 n$ lateral conditions are necessary for the problem to be well posed). The function $\omega$ can be defined as in (2.16) for $t \in[0, a]$; as

$$
\omega(t)=y^{1}(t)-y^{1}(b)+\int_{b}^{t}\left(a_{n-1}(s) y^{1}(s)+\cdots+a_{0}(s) y^{n}(s)\right) d s
$$

for $t \in[b, 1]$, and arbitrarily (continuous) on $] a, b[$.

2.3. An approximation result. We shall now state an easy approximation result (Proposition 2.4) that we will need partially in the proof of Proposition 4.8. Consider the space $C^{k}:=C^{k}\left([0,1] ; \mathbb{R}^{n \times n}\right)$, with $k$ a fixed nonnegative integer or $\infty$, endowed with its natural topology. Let $\mathcal{C}$ be the subset of $C^{k}$ comprising the matrix functions $A:[0,1] \rightarrow \mathbb{R}^{n \times n}$ of the form (2.5), with the topology induced by $C^{k}$. Fix a linear operator $\Lambda: C\left([0,1] ; \mathbb{R}^{n}\right) \rightarrow \mathbb{R}^{n}$ of rank $n$ and with the form given by (2.12), and consider the deterministic problems

$$
\begin{aligned}
& D Y(t)+A(t) Y(t)=0, \\
& \Lambda[Y]=0,
\end{aligned}
$$

with $A \in C^{k}$. Let $\mathscr{D} \subset C^{k}$ the class of matrix functions $A$ such that (2.17) has only the trivial solution. Finally set $V:=\mathcal{C} \cap \mathcal{D}$.

\section{LEMMA 2.3. $V$ is open and dense in $\mathrm{C}$.}

ProOF. For $A \in \mathcal{D}$, denote by $\Phi_{A}^{0}(t)$ the fundamental matrix solution of the system $D Y(t)+A(t) Y(t)=0$, with $Y(0)=\mathrm{I}$. Consider the composition of linear operators $\mathbb{R}^{n} \stackrel{\Gamma_{A}}{\rightarrow} C\left([0,1] ; \mathbb{R}^{n}\right) \stackrel{\Lambda}{\rightarrow} \mathbb{R}^{n}$ defined by

$$
c \mapsto\left[t \mapsto \Phi_{A}^{0}(t) c\right] \mapsto \Lambda\left[t \mapsto \Phi_{A}^{0}(t) c\right] .
$$

The mapping

$$
\begin{aligned}
C^{k} & \stackrel{\varphi}{\rightarrow} \mathcal{L}\left(\mathbb{R}^{n} ; \mathbb{R}^{n}\right), \\
A & \mapsto \Lambda \circ \Gamma_{A},
\end{aligned}
$$

is continuous. Indeed,

$$
\left\|\Lambda \circ \Gamma_{A}-\Lambda \circ \Gamma_{B}\right\|_{\mathcal{L}\left(\mathbb{R}^{n} ; \mathbb{R}^{n}\right)} \leq\|\Lambda\|_{\mathcal{L}\left(C, \mathbb{R}^{n}\right)} \cdot\left\|\Gamma_{A}-\Gamma_{B}\right\|_{\mathcal{L}\left(\mathbb{R}^{n}, C\right)}
$$


and

$$
\begin{aligned}
\left\|\Gamma_{A}-\Gamma_{B}\right\|_{\mathcal{L}\left(\mathbb{R}^{n}, C\right)} & =\sup _{\|c\|=1}\left\|\left(\Phi_{A}^{0}(t)-\Phi_{B}^{0}(t)\right) c\right\|_{\infty} \\
& =\max _{i, j} \sup _{t}\left|\Phi_{A}^{0}(t)_{i, j}-\Phi_{B}^{0}(t)_{i, j}\right| .
\end{aligned}
$$

The continuity follows from the uniform continuous dependence of the solution with respect to the data in a linear Cauchy problem.

Now we use the fact that the set $H$ of invertible operators on $\mathbb{R}^{n}$ is open in $\mathcal{L}\left(\mathbb{R}^{n}, \mathbb{R}^{n}\right)$. We obtain that $\varphi^{-1}(H)$ is open in $C^{k}$. However $\varphi^{-1}(H)$ is the set of matrices $A$ such that $\operatorname{det}\left(\Lambda \circ \Gamma_{A}\right) \neq 0$, which coincides with $\mathscr{D}$ by definition. This shows that $\mathscr{D}$ is open in $C^{k}$.

Since $\mathcal{C}$ is a linear manifold in $C^{k}$, we have that $V=\mathcal{C} \cap \mathcal{D}$ is open in $\mathcal{C}$. Note that the particular form of $\Lambda$ does not play any role up to this point.

To prove the density, we start by checking that $V \neq \varnothing$. If $A \in \mathcal{C}$ and $s \in[0,1]$, the corresponding fundamental matrix $\Phi_{A}^{S}(\cdot)$ has the form

$$
\Phi_{A}^{s}(t)=\left[\begin{array}{ccc}
D^{n-1} \phi_{1}(t) & \cdots & D^{n-1} \phi_{n}(t) \\
\vdots & & \vdots \\
D \phi_{1}(t) & \cdots & D \phi_{n}(t) \\
\phi_{1}(t) & \cdots & \phi_{n}(t)
\end{array}\right]
$$

with

$$
\Phi_{A}^{s}(s)=\mathrm{I}
$$

for some $C^{n+k}$ real functions $\phi_{1}, \ldots, \phi_{n}$. Conversely, any such matrix is the fundamental matrix solution $\Phi^{s}$ of $D Y(t)+A(t) Y(t)=0$ for some $A \in \mathcal{C}$. We have

$$
\int_{0}^{1} d F(t) \Phi^{s}(t)=\left[\begin{array}{ccc}
\alpha_{11} & \cdots & \alpha_{1 m} \\
\vdots & & \vdots \\
\alpha_{n 1} & \cdots & \alpha_{n m}
\end{array}\right]\left[\begin{array}{ccc}
\phi_{1}\left(t_{1}\right) & \cdots & \phi_{n}\left(t_{1}\right) \\
\vdots & & \vdots \\
\phi_{1}\left(t_{m}\right) & \cdots & \phi_{n}\left(t_{m}\right)
\end{array}\right]
$$

Take $s \notin\left\{t_{1}, \ldots, t_{m}\right\}$. Since $\left(\alpha_{i j}\right)$ has full rank and $n \leq m$, we can obviously find numbers $\phi_{i}\left(t_{j}\right), 1 \leq i \leq n, 1 \leq j \leq m$, such that this product is an invertible square matrix. Then we take $C^{n+k}$ functions $\phi_{1}, \ldots, \phi_{n}$ interpolating these numbers and so that $\left(D^{n-j} \phi_{i}(s)\right)_{i, j}=\mathrm{I}$. The corresponding $A$ will therefore belong to $V$.

Given now $A \in \mathscr{D}$, let us fix $A_{0} \in V$. For $\lambda \in \mathbb{R}$, define

$$
M_{\lambda}:=\left[\begin{array}{cccc}
0 & 0 & \cdots & 0 \\
-1 & 0 & \cdots & 0 \\
\vdots & \ddots & \ddots & \vdots \\
0 & \cdots & -1 & 0
\end{array}\right]+\left[\begin{array}{cccc}
\lambda & 0 & \cdots & 0 \\
0 & 0 & \cdots & 0 \\
\vdots & \vdots & \ddots & \vdots \\
0 & 0 & \cdots & 0
\end{array}\right] A_{0}+\left[\begin{array}{cccc}
1-\lambda & 0 & \cdots & 0 \\
0 & 0 & \cdots & 0 \\
\vdots & \vdots & \ddots & \vdots \\
0 & 0 & \cdots & 0
\end{array}\right] A
$$


Let us see that the function $u: \lambda \mapsto \operatorname{det} \Lambda\left[\Phi_{M_{\lambda}}^{0}\right]$ is analytical: indeed, $M_{\lambda}$ depends analytically on $\lambda$, and so the fundamental solution $\Phi_{M_{\lambda}}^{0}$ is also analytic in $\lambda$. Finally, analyticity is preserved by the linear functional $\Lambda$ and the determinant. Now assume $u \equiv 0$ in a neighborhood of 0 . This would imply $u \equiv 0$ on the whole line. However,

$$
u(1)=\operatorname{det} \Lambda\left[\Phi_{A_{0}}^{0}\right] \neq 0 .
$$

We conclude that there exists a sequence $\left\{\lambda_{n}\right\}_{n}$ converging to zero such that $M_{\lambda_{n}} \in V$. Since $M_{\lambda_{n}} \rightarrow A$ as $n \rightarrow \infty$, the density is proved.

This proof borrows some ideas from Theorem 7.1 in [6].

Proposition 2.4. Let $A^{N}(t)$ be a sequence of functions in $V$ converging to $A(t) \in V$. Let $Y^{N}(t)$ and $Y(t)$ be the corresponding unique solutions to

$$
\begin{aligned}
& D Y^{N}(t)+A^{N}(t) Y^{N}(t)=\dot{W}(t), \quad \text { and } \quad D Y(t)+A(t) Y(t)=\dot{W}(t), \\
& \Lambda[Y]=c, \quad \Lambda[Y]=c \text {. }
\end{aligned}
$$

Then $Y^{N}(t)$ converges to $Y(t)$ pointwise and in $L^{p}$, for all $p \geq 1$, uniformly in $t$, that is,

$$
\begin{aligned}
& \lim _{N \rightarrow+\infty} \sup _{0 \leq t \leq 1}\left|Y^{N}(t)(\omega)-Y(t)(\omega)\right|=0 \quad \forall \omega \in \Omega, \\
& \lim _{N \rightarrow+\infty} \sup _{0 \leq t \leq 1}\left\|Y^{N}(t)-Y(t)\right\|_{L^{p}(\Omega)}=0 .
\end{aligned}
$$

Proof. In the situation given, the fundamental solutions $\Phi^{t}(u)^{N}$ converge to the fundamental solution $\Phi^{t}(u)$ uniformly in $t$ and $u$. From this fact one shows easily that $J^{N}$ and $\left(J^{N}\right)^{-1}$ defined by (2.9) converge uniformly to $J$ and $J^{-1}$, taking into account that the entries of $d F$ are finite measures. Hence, the Green functions $G^{N}(t, s)$ converge to $G(t, s)$ uniformly in $t$ and $s$ as well.

We have

$$
\left\|Y^{N}(t)-Y(t)\right\|_{L^{p}} \leq\left|J^{N}(t)^{-1} c-J(t)^{-1} c\right|+\left\|\int_{0}^{1}\left(G^{N}(t, s)-G(t, s)\right) d B_{s}\right\|_{L^{p}} .
$$

The first term tends to zero uniformly in $t$. For the second, note that

$$
\begin{aligned}
& \sup _{t} \mathrm{E} {\left[\left(\int_{0}^{1}\left(G_{i, j}^{N}(t, s)-G_{i, j}(t, s)\right) d W_{s}\right)^{2}\right] } \\
&=\sup _{t} \int_{0}^{1}\left(G_{i, j}^{N}(t, s)-G_{i, j}(t, s)\right)^{2} d s \\
& \leq \int_{0}^{1} \sup _{t}\left(G_{i, j}^{N}(t, s)-G_{i, j}(t, s)\right)^{2} d s \\
& \leq \sup _{t} \sup _{s}\left(G_{i, j}^{N}(t, s)-G_{i, j}(t, s)\right)^{2},
\end{aligned}
$$


which converges to zero. Since all random variables are Gaussian, the convergence to zero of the second moments (uniformly in $t$ ) implies the convergence to zero of all moments, also uniformly in $t$. We have proved the second statement of the theorem.

We turn to the pointwise convergence: Since $G_{i, j}^{N}(t, s)-G_{i, j}(t, s)$ is a function of bounded variation which tends to zero uniformly in $t$ and $s$, the finite measures $G_{i, j}^{N}(t, d s)-G_{i, j}(t, d s)$ tend weakly to zero, uniformly in $t$, and we have

$$
\begin{aligned}
& \left|\int_{0}^{1}\left(G_{i, j}^{N}(t, s) d W_{s}-G_{i, j}(t, s) d W_{s}\right)\right| \\
& \quad=\left|\int_{0}^{1} W_{s} \cdot\left(G_{i, j}^{N}(t, d s)-G_{i, j}(t, d s)\right)\right| \rightarrow 0 .
\end{aligned}
$$

3. A characterization of conditional independence and convergence of conditional expectations. In this section we state two facts of a general nature that will be our main probabilistic tools in Section 4. Lemma 3.4 is an abstract result on the conditional independence of two random vectors when a function of them (of a special structure) is given; it was proved in [2] (see also [9]). Proposition 3.7, on the other hand, provides a sufficient condition for the $L^{2}$-convergence as $N \rightarrow \infty$ of a sequence of conditional expectations of the form $\mathrm{E}\left[F\left(U_{1}^{N}\right) \mid U_{2}^{N}\right]$.

We will mention first three auxiliary lemmas on the conditional independence of $\sigma$-fields, whose proofs are not difficult. Recall that we write $\mathcal{F}_{1} \Perp_{g} \mathcal{F}_{2}$ to mean that the $\sigma$-fields $\mathcal{F}_{1}$ and $\mathscr{F}_{2}$ are conditionally independent given the $\sigma$-field $g$.

LEMMA 3.1. Let $\mathcal{F}_{1}, \mathcal{F}_{2}, \mathcal{G}, \mathcal{F}_{1}^{\prime}, \mathcal{F}_{2}^{\prime}$ be $\sigma$-fields such that $\mathcal{F}_{1}^{\prime} \subset \mathcal{F}_{1} \vee g$ and $\mathcal{F}_{2}^{\prime} \subset \mathcal{F}_{2} \vee \mathcal{g}$. Then,

$$
\mathcal{F}_{1} \frac{\Perp}{g} \mathcal{F}_{2} \Longrightarrow \mathcal{F}_{1}^{\prime} \frac{\Perp}{g} \mathcal{F}_{2}^{\prime}
$$

LEMMA 3.2. Let $\mathcal{F}_{1}, \mathcal{F}_{2}, \mathcal{F}_{3}$ and $g$ be $\sigma$-fields such that $\mathcal{F}_{1} \vee \mathcal{F}_{2} \Perp_{g} \mathcal{F}_{3}$ and $\mathcal{F}_{1} \Perp_{g} \mathcal{F}_{2}$. Then,

$$
\mathcal{F}_{1} \vee \mathcal{F}_{3} \frac{\Perp}{g} \mathcal{F}_{2} \quad \text { and } \quad \mathcal{F}_{2} \vee \mathcal{F}_{3} \frac{\Perp}{g} \mathcal{F}_{1}
$$

LEMMA 3.3. Let $\mathcal{F}_{1}, \mathcal{F}_{2}$ and $g$ be $\sigma$-fields such that $\mathcal{F}_{1} \Perp_{g} \mathscr{F}_{2}$ and $\mathcal{g} \subset \mathcal{F}_{1}$. Then, for any $\sigma$-field $\mathscr{H}$, with $\mathscr{G} \subset \mathscr{H} \subset \mathcal{F}_{1}$,

$$
\mathcal{F}_{1} \frac{\Perp}{\mathscr{H}} \mathcal{F}_{2} \text {. }
$$

Let $(\Omega, \mathcal{F}, P)$ be a probability space and $\mathcal{F}_{1}$ and $\mathcal{F}_{2}$ two independent sub$\sigma$-fields of $\mathcal{F}$. Consider two functions $g_{1}: \mathbb{R}^{d} \times \Omega \rightarrow \mathbb{R}^{d}$ and $g_{2}: \mathbb{R}^{d} \times \Omega \rightarrow \mathbb{R}^{d}$ 
such that $g_{i}$ is $\mathscr{B}\left(\mathbb{R}^{d}\right) \otimes \mathcal{F}_{i}$-measurable, $i=1$, 2. Set $B(\varepsilon):=\left\{x \in \mathbb{R}^{d},|x|<\varepsilon\right\}$, and denote by $\lambda$ the Lebesgue measure on $\mathbb{R}^{d}$. Let us introduce the following hypotheses:

(H1) There exists $\varepsilon_{0}>0$ such that for almost all $\omega \in \Omega$, and for any $|\xi|<\varepsilon_{0}$, $|\eta|<\varepsilon_{0}$, the system

$$
\begin{aligned}
& z_{1}-g_{1}\left(z_{2}, \omega\right)=\xi, \\
& z_{2}-g_{2}\left(z_{1}, \omega\right)=\eta
\end{aligned}
$$

has a unique solution $\left(z_{1}, z_{2}\right) \in \mathbb{R}^{2 d}$.

(H2) For every $z_{1} \in \mathbb{R}^{d}$ and $z_{2} \in \mathbb{R}^{d}$, the random vectors $g_{1}\left(z_{2}, \cdot\right)$ and $g_{2}\left(z_{1}, \cdot\right)$ possess absolutely continuous distributions and the function

$$
\delta\left(z_{1}, z_{2}\right)=\sup _{0<\varepsilon<\varepsilon_{0}} \frac{1}{\lambda(B(\varepsilon))^{2}} P\left\{\left|z_{1}-g_{1}\left(z_{2}\right)\right|<\varepsilon ;\left|z_{2}-g_{2}\left(z_{1}\right)\right|<\varepsilon\right\}
$$

is locally integrable in $\mathbb{R}^{2 d}$, for some $\varepsilon_{0}>0$.

(H3) For almost all $\omega \in \Omega$, the functions $z_{2} \mapsto g_{1}\left(z_{2}, \omega\right)$ and $z_{1} \mapsto g_{2}\left(z_{1}, \omega\right)$ are continuously differentiable and

$$
\sup _{\left|z_{2}-g_{2}\left(z_{1}, \omega\right)\right|<\varepsilon_{0},\left|z_{1}-g_{1}\left(z_{2}, \omega\right)\right|<\varepsilon_{0}}\left|\operatorname{det}\left[\mathrm{I}-\nabla g_{1}\left(z_{2}, \omega\right) \nabla g_{2}\left(z_{1}, \omega\right)\right]\right|^{-1} \in L^{1}(\Omega)
$$

for some $\varepsilon_{0}>0$, where $\nabla g_{i}$ denotes the Jacobian matrix of $g_{i}$ with respect to the first argument.

Note that hypothesis (H1) implies the existence of two random vectors $Z_{1}$ and $Z_{2}$ determined by the system

$$
\begin{aligned}
& Z_{1}(\omega)=g_{1}\left(Z_{2}(\omega), \omega\right), \\
& Z_{2}(\omega)=g_{2}\left(Z_{1}(\omega), \omega\right) .
\end{aligned}
$$

Lemma 3.4 ([2]). Suppose the functions $g_{1}$ and $g_{2}$ satisfy the above hypotheses $(\mathrm{H} 1)-(\mathrm{H} 3)$. Then the following statements are equivalent:

(i) $\mathcal{F}_{1}$ and $\mathcal{F}_{2}$ are conditionally independent given the random vectors $Z_{1}, Z_{2}$.

(ii) There exist two functions $F_{i}: \mathbb{R}^{2 d} \times \Omega \rightarrow \mathbb{R}, i=1,2$, which are $\mathcal{B}\left(\mathbb{R}^{2 d}\right) \otimes$ $\mathcal{F}_{i}$-measurable, such that

$$
\left|\operatorname{det}\left[\mathrm{I}-\nabla g_{1}\left(Z_{2}\right) \nabla g_{2}\left(Z_{1}\right)\right]\right|=F_{1}\left(Z_{1}, Z_{2}, \omega\right) F_{2}\left(Z_{1}, Z_{2}, \omega\right) \quad \text { a.s. }
$$

Goggin [10] gives a sufficient condition for the convergence in distribution of a sequence of conditional expectations of the form $\mathrm{E}\left[F\left(U_{1}^{N}\right) \mid U_{2}^{N}\right]$. We reproduce here a slightly simplified version. Combining this result with Lemma 3.6, due to Knudsen [13], we can easily prove our Proposition 3.7. 
LEMMA 3.5 ([10]). Let $U_{1}^{N}$ and $U_{2}^{N}$ be two sequences of random vectors on a probability space $(\Omega, \mathcal{F}, \mathrm{P})$, such that $\left(U_{1}^{N}, U_{2}^{N}\right) \rightarrow\left(U_{1}, U_{2}\right)$ as $N \rightarrow \infty$ in distribution. Assume that:

(i) There exist probabilities $Q^{N}$ on $(\Omega, \mathcal{F})$ such that $P \ll Q^{N}$ on $\sigma\left\{U_{1}^{N}\right.$, $\left.U_{2}^{N}\right\}$ and $U_{1}^{N}$ and $U_{2}^{N}$ are independent under $Q^{N}$. Denote $\ell^{N}\left(U_{1}^{N}, U_{2}^{N}\right):=\frac{d P}{d Q^{N}}$.

(ii) There exists a probability $Q$ on $(\Omega, \mathcal{F})$ under which $U_{1}$ and $U_{2}$ are independent.

(iii) The $Q^{N}$-distribution of $\left(U_{1}^{N}, U_{2}^{N}, \ell^{N}\left(U_{1}^{N}, U_{2}^{N}\right)\right)$ converges weakly to the $Q$-distribution of $\left(U_{1}, U_{2}, \ell\left(U_{1}, U_{2}\right)\right)$, where $\ell$ is such that $\mathrm{E}_{Q}\left[\ell\left(U_{1}, U_{2}\right)\right]=1$.

Then:

(i) $P \ll Q$ on $\sigma\left\{U_{1}, U_{2}\right\}$ and $\frac{d P}{d Q}=\ell\left(U_{1}, U_{2}\right)$.

(ii) For every bounded continuous function $F$,

$$
\mathrm{E}_{P}\left[F\left(U_{1}^{N}\right) \mid U_{2}^{N}\right] \rightarrow \mathrm{E}_{P}\left[F\left(U_{1}\right) \mid U_{2}\right] \quad \text { in distribution. }
$$

LEMMA 3.6 ([13]). Let $U_{1}^{N}$ and $U_{2}^{N}$ be two sequences of random vectors on a probability space $(\Omega, \mathcal{F}, \mathcal{P})$. Assume that, as $N \rightarrow \infty$ :

(i) $U_{2}^{N} \stackrel{P}{\rightarrow} U_{2}$.

(ii) $U_{1}^{N} \stackrel{L^{2}}{\rightarrow} U_{1}$, with $U_{1} \in L^{p}$, for some $p>2$.

(iii) $\left\|\mathrm{E}\left[U_{1} \mid U_{2}^{N}\right]\right\|_{L^{2}} \rightarrow\left\|\mathrm{E}\left[U_{1} \mid U_{2}\right]\right\|_{L^{2}}$.

Then

$$
\mathrm{E}\left[U_{1}^{N} \mid U_{2}^{N}\right] \stackrel{L^{2}}{\rightarrow} \mathrm{E}\left[U_{1} \mid U_{2}\right]
$$

as $N \rightarrow \infty$.

Combining Lemmas 3.5 and 3.6, we get the following proposition.

Proposition 3.7. Let $U_{1}^{N}$ and $U_{2}^{N}$ be two sequences of random vectors. Assume that, as $N \rightarrow \infty$ :

(i) $U_{1}^{N} \stackrel{P}{\rightarrow} U_{1}$ and $U_{2}^{N} \stackrel{P}{\rightarrow} U_{2}$.

(ii) Hypotheses 1-3 of Lemma 3.5 hold true.

Then, for any bounded and continuous function $F$,

$$
\mathrm{E}\left[F\left(U_{1}^{N}\right) \mid U_{2}^{N}\right] \stackrel{L^{2}}{\rightarrow} \mathrm{E}\left[F\left(U_{1}\right) \mid U_{2}\right] .
$$

PROOF. Applying Lemma 3.5, we have that, for every bounded and continuous $F$,

$$
\mathrm{E}\left[F\left(U_{1}^{N}\right) \mid U_{2}^{N}\right] \rightarrow \mathrm{E}\left[F\left(U_{1}\right) \mid U_{2}\right] \quad \text { in distribution. }
$$


From (3.1) and the fact that $F\left(U_{1}^{N}\right), F\left(U_{1}\right) \in L^{\infty}$, we obtain the convergence of the $L^{2}$ norms:

$$
\left\|\mathrm{E}\left[F\left(U_{1}^{N}\right) \mid U_{2}^{N}\right]\right\|_{L^{2}} \rightarrow\left\|\mathrm{E}\left[F\left(U_{1}\right) \mid U_{2}\right]\right\|_{L^{2}} .
$$

On the other hand, since $F$ is bounded and $U_{1}^{N} \stackrel{P}{\rightarrow} U_{1}$, we also have

$$
F\left(U_{1}^{N}\right) \stackrel{L^{2}}{\rightarrow} F\left(U_{1}\right) .
$$

Now, (3.2) and (3.3) imply that

$$
\left\|\mathrm{E}\left[F\left(U_{1}\right) \mid U_{2}^{N}\right]\right\|_{L^{2}} \rightarrow\left\|\mathrm{E}\left[F\left(U_{1}\right) \mid U_{2}\right]\right\|_{L^{2}}
$$

and we get the conclusion applying Lemma 3.6.

4. Markovian properties of linear functional boundary value problems. In the study of boundary value stochastic problems, one of the main interests has been to seek conditions on the coefficients for the solution process to satisfy some suitably defined Markov-type property. Intuition suggests that a relation $h(X(0), X(1))=0$ will possibly prevent the Markov process property from holding in general. One might think that nevertheless the Markov field property, which is defined below, will be satisfied. It is easy to see that any Markov process is a Markov field (see [12] for the continuous case and [3] for a simple proof in the general case). The converse is not true. For instance, the processes $X(t)=W(t)-\alpha W(1)$ are Markov fields; they are not Markov processes, except for the cases $\alpha=0$ and $\alpha=1$.

DEFinition 4.1. A process $\{X(t), t \in[0,1]\}$ is said to be a Markov field if for any $0 \leq a<b \leq 1$, the $\sigma$-fields $\sigma\{X(t), t \in[a, b]\}$ and $\sigma\{X(t), t \in] a, b\left[{ }^{c}\right\}$ are conditionally independent given $\sigma\{X(a), X(b)\}$.

However, even this weaker property holds only in special cases. For instance, in [2] it was shown that the solution to

$$
\begin{aligned}
& \dot{X}(t)=b(X(t))+\sigma(X(t)) \circ \dot{W}(t), \quad t \in[0,1], \\
& X(0)=\psi(X(1)),
\end{aligned}
$$

where the stochastic integral is understood in the Stratonovich sense, is a Markov field if and only if $b(x)=A \sigma(x)+B \sigma(x) \int_{c}^{x} \frac{1}{\sigma(t)} d t$, for some constants $A, B, c$. As a corollary, in case $\sigma$ is a constant (additive noise), $X$ is a Markov field if and only if $b$ is an affine function.

Our aim is to study the linear-additive case when the additional condition takes into account the value of the solution in some interior points of the time interval. The following simple example illustrates that the situation changes. 
EXAMPLE 4.2. Consider the first-order system

$$
\begin{aligned}
& \dot{X}(t)=\dot{W}(t), \quad t \in[0,1], \\
& X\left(\frac{1}{2}\right)+X(1)=0 .
\end{aligned}
$$

The solution is the process

$$
X(t)=-\frac{1}{2}\left(W\left(\frac{1}{2}\right)+W(1)\right)+W(t),
$$

which is not a Markov field. Indeed, for $a=0$ and $b=\frac{2}{3}$, the random variables $X\left(\frac{1}{2}\right)$ and $X(1)$ are not conditionally independent given $\sigma\{X(a), X(b)\}$. Nevertheless, $X$ is a Markov field when restricted to $\left[0, \frac{1}{2}\right]$ or $\left[\frac{1}{2}, 1\right]$.

In order to formulate precisely the conditional independence property enjoyed by the system (2.3) and (2.4), we introduce first some more concepts and notation.

Let $\Lambda_{1}, \ldots, \Lambda_{n}$ be the real-valued components of a boundary operator $\Lambda$ of the form (2.4) and denote their support by $\operatorname{supp} \Lambda_{i}:=\left\{t_{j} \in[0,1]: \alpha_{i j} \neq 0\right\}$.

Definition 4.3. We will say that $\Lambda_{i}$ preserves the pair $(a, b)$ if either $\left.\operatorname{supp} \Lambda_{i} \subset\right] a, b\left[\right.$ or $\operatorname{supp} \Lambda_{i} \subset[a, b]^{c}$. If this is true for all $i$ (i.e., there are no boundary conditions involving simultaneously points inside and outside $[a, b]$ ), then we will also say that $\Lambda$ preserves $(a, b)$.

We want to prove that the solution $Y$ to the system (2.3) and (2.4) satisfies the following conditional independence property (Theorem 4.5): If $\Lambda$ preserves $(a, b)$, then

$$
\sigma\{Y(t), t \in[a, b]\} \underset{\sigma\{Y(a), Y(b)\}}{\frac{1}{(Y)}} \sigma\{(t), t \in] a, b\left[^{c}\right\} .
$$

More generally, this conditional independence is also true when $\Lambda$ does not preserve $(a, b)$, provided the conditioning $\sigma$-field is enlarged with the variables $Y_{n}(t)$, for $t$ in $[a, b]$ and in the support of all nonpreserving boundary operators $\Lambda_{i}$ (Theorem 4.10).

Since the boundary conditions can be written in many different equivalent ways, and the sets supp $\Lambda_{i}$ (hence the property of preserving an interval) depend on the representation chosen, we need, before proceeding further, some sort of "canonical" definition of the linear operator $\Lambda$. Given supp $\Lambda=\left\{t_{1}, \ldots, t_{m}\right\}, \Lambda$ can be regarded as a linear mapping $\mathbb{R}^{m} \rightarrow \mathbb{R}^{n}$, that means, an $n \times m$ matrix acting on the vector $\left(Y_{n}\left(t_{1}\right), \ldots, Y_{n}\left(t_{m}\right)\right)$ (see the notation in Section 2 ).

A basis $B$ for an $n \times m$ matrix $\Lambda$ is any $n \times n$ minor with full rank. For notational simplicity, assume that $B$ consists of the firsts $n$ columns of $\Lambda$. Denoting by $N$ the nonbasic columns, we can write $\Lambda=(B, N)$. Defining $\widetilde{\Lambda}=\left(\mathrm{I}, B^{-1} N\right)$, the system of equations $\Lambda x=c$ can be written in the equivalent form $\widetilde{\Lambda} x=B^{-1} c$. In this situation, we shall say that $\tilde{\Lambda}$ is a basic expression of $\Lambda$ relative to 
the basis $B$. In the following lemma we prove that this representation can be considered "canonical" for our purposes, since any pair $(a, b)$ will or will not be preserved by any basic equivalent form of $\Lambda$. In the sequel, we will always assume, without explicit mention, that the boundary condition is written in this form.

LEMMA 4.4. Let $\tilde{\Lambda}$ and $\widetilde{\Lambda}^{\prime}$ be two basic expressions of $\Lambda$, and fix $0 \leq a<$ $b \leq 1$. Then, $\tilde{\Lambda}$ preserves $(a, b)$ if and only if $\tilde{\Lambda}^{\prime}$ preserves $(a, b)$.

PROOF. Without any loss of generality we can assume that $\widetilde{\Lambda}=(\mathrm{I}, N)$, where $\mathrm{I}$ is the $n \times n$ identity matrix and

$$
N=\left[\begin{array}{ccc}
\alpha_{1, n+1} & \cdots & \alpha_{1, m} \\
\vdots & \ddots & \vdots \\
\alpha_{n, n+1} & \cdots & \alpha_{n, m}
\end{array}\right]
$$

All basic expressions of the original matrix $\Lambda$ can be obtained by repeated Gaussian pivoting on entries of nonbasic columns; when pivoting on $\alpha_{i k}$, the column $i$ leaves the basis (the identity matrix) and is replaced by column $k$. Therefore, it is sufficient to prove the lemma for $\tilde{\Lambda}$ and a basic expression $\tilde{\Lambda}^{\prime}$ obtained from $\tilde{\Lambda}$ by one pivoting operation.

Let us assume that $\alpha_{1, n+1} \neq 0$ and that the operator $\widetilde{\Lambda}_{i}$ does not preserve the pair $(a, b)$. We are going to find an operator $\tilde{\Lambda}_{j}^{\prime}$ which neither preserves $(a, b)$. Rows 1 and $i$ before and after pivoting on $\alpha_{1, n+1}$ are the following:

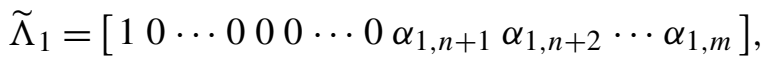

$$
\begin{aligned}
& \widetilde{\Lambda}_{i}=\left[\begin{array}{lllllllll}
0 & 0 & \cdots & 0 & 1 & 0 & \cdots & 0 & \alpha_{i, n+1} \\
\alpha_{i, n+2} & \cdots & \alpha_{i, m}
\end{array}\right], \\
& \widetilde{\Lambda}_{1}^{\prime}=\left[\frac{1}{\alpha_{1, n+1}} 0 \cdots 0000001 \frac{\alpha_{1, n+2}}{\alpha_{1, n+1}} \cdots \frac{\alpha_{1, m}}{\alpha_{1, n+1}}\right] \text {, }
\end{aligned}
$$

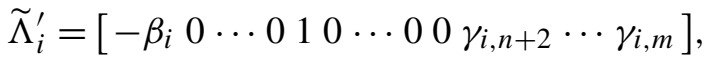

where $\beta_{i}=\frac{\alpha_{i, n+1}}{\alpha_{1, n+1}}$ and $\gamma_{i, j}=\alpha_{i, j}-\alpha_{1, j} \beta_{i}$.

If $\widetilde{\Lambda}_{1}$ does not preserve $(a, b)$, then the result is trivially true, with $j=1$, since $\widetilde{\Lambda}_{1}$ and $\widetilde{\Lambda}_{1}^{\prime}$ have their nonzero coefficients in the same columns. By a similar reason, if $\beta_{i}=0$ we can take $j=i$.

Assume finally that $\left.\operatorname{supp} \widetilde{\Lambda}_{1} \subset\right] a, b\left[\right.$ (so that $i \neq 1$ ), and that $\beta_{i} \neq 0$. If $t_{i} \in$ $[a, b]^{c}$, the result is proved, since $\widetilde{\Lambda}_{i}^{\prime}$ links $t_{1}$ and $t_{i}$, and $\left.t_{1} \in\right] a, b[$; take $j=i$. If $\left.t_{i} \in\right] a, b\left[\right.$, then there exists $k \in\{n+1, \ldots, m\}$ such that $t_{k} \in[a, b]^{c}$ with $\alpha_{i k} \neq 0$; since $\alpha_{1 k}=0$ (because supp $\left.\widetilde{\Lambda}_{1} \subset\right] a, b\left[\right.$ ), we have $\gamma_{i k}=\alpha_{i k} \neq 0$ and we can take again $j=i$.

Now we can formulate our main result: 
THEOREM 4.5. Suppose the system

$$
\begin{aligned}
& D Y(t)+A(t) Y(t)=\dot{B}(t), \quad t \in[0,1], \\
& \Lambda[Y]=c,
\end{aligned}
$$

satisfies $(\mathrm{H} 0)$, and let $Y=\{Y(t), t \in[0,1]\}$ be its unique solution. Then,

$$
\sigma\{Y(t), t \in[a, b]\} \underset{\sigma\{Y(a), Y(b)\}}{\Perp} \sigma\{Y(t), t \in] a, b\left[^{c}\right\}
$$

if and only if the pair $(a, b)$ is preserved by $\Lambda$.

Our main tool for the proof of the "if" part in Theorem 4.5 will be Lemma 3.4. The idea is the following: We will split the $2 n$-dimensional random vector $(Y(a), Y(b))$ into two vectors $Z^{1}$ and $Z^{2}$ of suitable dimensions, in such a way that $Z^{1}$ be a function of $Z^{2}$ and the increments of the Wiener process $W$ in $[a, b]$, and in turn $Z^{2}$ be a function of $Z^{1}$ and the increments of $W$ in $] a, b{ }^{c}$. These mappings will play the role of $g_{1}$ and $g_{2}$ in the set of hypotheses (H1) to (H3). The first will be defined through the solution to equation $D Y(t)+A(t) Y(t)=\dot{B}(t)$, with the components of $Z^{2}$ fixed to a constant; the second will be defined similarly, fixing the components of $Z^{1}$ to a constant. However, this means that we need to solve our differential equation with several sets of constraints, which are different from the original set, and therefore we cannot ensure a priori that these problems are well posed. Consequently, the above functions $g_{1}$ and $g_{2}$ need not exist in general.

To solve this technical difficulty, we will resort to a two-step procedure. First, we will assume that all functional boundary value problems that we need to solve are indeed well posed. Then, hypotheses $(\mathrm{H} 1)$ to $(\mathrm{H} 3)$ can be checked, and Lemma 3.4 applies directly, yielding the desired result. This is the goal of Proposition 4.7. Secondly, we will use the approximation result of Section 2.3 to show that the matrix $A$ can be approximated by perturbed matrices $A^{N}$ for which all boundary problems involved are well posed and whose solutions $Y^{N}$ converge to the solution $Y$ of the original problem. Then, the convergence of conditional expectations given in Proposition 3.7 will allow to carry the conditional independence properties of $Y^{N}$ to the limit. This second step is the contents of Proposition 4.8. The "only if" part of the theorem is shown in Proposition 4.9.

Let us formulate precisely the assumption needed for the first step. Set

$$
\begin{aligned}
& \ell=\#\left\{i: \operatorname{supp} \Lambda_{i} \subset[0, a[\},\right. \\
& \left.\left.p=\#\left\{i: \operatorname{supp} \Lambda_{i} \subset\right] b, 1\right]\right\}, \\
& q=\#\left\{i: \operatorname{supp} \Lambda_{i} \subset\right] a, b[\} .
\end{aligned}
$$

We can assume that the equalities $\Lambda_{i}[X]=c_{i}$ are ordered in the following way:

$$
\begin{array}{ll}
\operatorname{supp} \Lambda_{i} \subset[0, a[, & i=1, \ldots, \ell, \\
\left.\operatorname{supp} \Lambda_{i} \subset\right] a, b[, & i=\ell+1, \ldots, \ell+q, \\
\left.\left.\operatorname{supp} \Lambda_{i} \subset\right] b, 1\right], & i=\ell+q+1, \ldots, \ell+q+p,
\end{array}
$$


and the remaining equations (those involving points both in $[0, a[$ and in $] b, 1]$ ), carry the labels $i=\ell+q+p+1, \ldots, n$.

Consider now $D Y(t)+A(t) Y(t)=0$ with the following sets of lateral conditions and the specified domain:

$$
\begin{aligned}
& Y_{j}(a)=0, j=1, \ldots, n-\ell, \\
& \Lambda_{i}[Y]=0, i=1, \ldots, \ell, \\
& Y_{j}(b)=0, j=1, \ldots, n-\ell-q, \\
& Y_{j}(a)=0, j=n-\ell+1, \ldots, n, \quad \text { on }[0, a], \\
& \Lambda_{i}[Y]=0, i=\ell+1, \ldots, \ell+q, \\
& Y_{j}(b)=0, j=n-\ell-q+1, \ldots, n, \\
& \Lambda_{i}[Y]=0, i=\ell+q+1, \ldots, \ell+q+p, \\
& \Lambda_{i}[Y]=0, i=\ell+q+p+1, \ldots, n, \\
& Y_{n}(t)=0 \quad \forall t \in \quad \bigcup_{i=\ell+q+p+1}^{n}\left(\operatorname{supp} \Lambda_{i} \cap[0, a]\right),
\end{aligned}
$$

(notice that the third and fourth lines result in $n-\ell-q-p$ equations involving only points in $[b, 1])$.

Definition 4.6. We will say that the pair $(a, b)$ is regular if $D Y(t)+$ $A(t) Y(t)=0$ together with any of the sets of conditions (4.5), (4.6) or (4.7) has only the trivial solution. Otherwise $(a, b)$ will be called singular.

\section{Proposition 4.7. Suppose the system}

$$
\begin{aligned}
& D Y(t)+A(t) Y(t)=\dot{B}(t), \quad t \in[0,1], \\
& \Lambda[Y]=c
\end{aligned}
$$

satisfies (H0), and let $Y=\{Y(t), t \in[0,1]\}$ be its unique solution. Let $(a, b)$ be a regular pair preserved by $\Lambda$. Then (4.2) holds true.

Proof. Let us define the $\sigma$-fields:

$$
\begin{aligned}
& \mathcal{F}_{a, b}^{i}=\sigma\left\{W_{t}-W_{a}, t \in[a, b]\right\}, \\
& \mathcal{F}_{a, b}^{e}=\sigma\left\{W_{t}, t \in[0, a]\right\} \vee \sigma\left\{W_{1}-W_{t}, t \in[b, 1]\right\}
\end{aligned}
$$

for $0 \leq a<b \leq 1$. Notice that $\mathcal{F}_{a, b}^{i}$ and $\mathcal{F}_{a, b}^{e}$ are independent.

We shall divide the proof into several steps. In Step 1 we reduce the proof to that of the conditional independence of two independent $\sigma$-fields. In Step 2 it is 
shown that there exist the two functions $g_{1}$ and $g_{2}$ needed to apply Lemma 3.4. The hypotheses of this lemma are checked in Steps 3, 4 and 5. In Step 6 we finally conclude the result.

STEP 1. Denote $g_{a, b}=\sigma\{Y(a), Y(b)\}$. If

$$
\mathcal{F}_{a, b}^{i} \frac{\|}{g_{a, b}} \mathcal{F}_{a, b}^{e},
$$

then (4.2) holds.

PROOF OF STEP 1. It is immediate to prove that $\sigma\{Y(t), t \in[a, b]\} \subset$ $g_{a, b} \vee \mathcal{F}_{a, b}^{i}$, and $\sigma\{Y(t), t \in] a, b\left[{ }^{c}\right\} \subset G_{a, b} \vee \mathcal{F}_{a, b}^{e}$. We apply then Lemma 3.1.

STEP 2. Let $\ell, p$ and $q$ be as in (4.3). We will denote by $\widetilde{Y}$ the solution to (4.8), to distinguish the actual solution from $Y$ regarded as an unknown of the system. Define

$$
\begin{aligned}
& Z^{1}:=\left(\widetilde{Y}_{1}(a), \ldots, \widetilde{Y}_{n-\ell}(a), \widetilde{Y}_{n-\ell-q+1}(b), \ldots, \widetilde{Y}_{n}(b)\right) \in \mathbb{R}^{n+q}, \\
& Z^{2}:=\left(\widetilde{Y}_{1}(b), \ldots, \widetilde{Y}_{n-\ell-q}(b), \widetilde{Y}_{n-\ell+1}(a), \ldots, \widetilde{Y}_{n}(a)\right) \in \mathbb{R}^{n-q} .
\end{aligned}
$$

Then, there exist two functions

$$
\begin{aligned}
& g_{1}: \mathbb{R}^{n-q} \times \Omega \rightarrow \mathbb{R}^{n+q}, \\
& g_{2}: \mathbb{R}^{n+q} \times \Omega \rightarrow \mathbb{R}^{n-q},
\end{aligned}
$$

measurable with respect to $\mathcal{B}\left(\mathbb{R}^{n-q}\right) \otimes \mathcal{F}_{a, b}^{i}$ and $\mathcal{B}\left(\mathbb{R}^{n+q}\right) \otimes \mathcal{F}_{a, b}^{e}$, respectively, and such that

$$
Z^{1}=g_{1}\left(Z^{2}, \omega\right) \quad \text { and } \quad Z^{2}=g_{2}\left(Z^{1}, \omega\right) .
$$

ProOF OF STEP 2. Consider the lateral conditions

$$
\begin{aligned}
Y_{j}(b) & =Z_{j}^{2}, & j & =1, \ldots, n-\ell-q, \\
Y_{j}(a) & =Z_{j-q}^{2}, & j & =n-\ell+1, \ldots, n, \\
\Lambda_{i}[Y] & =c_{i}, & i & =\ell+1, \ldots, \ell+q,
\end{aligned}
$$

on $[a, b]$. The process $\tilde{Y}$ trivially satisfies $D Y(t)+A(t) Y(t)=\dot{B}(t)$ and these conditions on $[a, b]$; however the solution to this problem is also unique. Therefore, taking into account that $\Lambda_{i}[\tilde{Y}]=c_{i}$ are constants, the vector $Z^{1}$ is determined by $Z^{2}$ and the increments of the Wiener process in $[a, b]$. Moreover, the function $g_{1}\left(z_{2}, \omega\right)$ so defined has a sense for every $z_{2} \in \mathbb{R}^{n-q}$, because we have that the solution to (2.3) - (4.11) is unique, and this fact does not depend on the particular right-hand sides. 
We want to prove analogously the existence of the function $g_{2}$. Consider first $D Y(t)+A(t) Y(t)=\dot{B}(t)$ on $[0, a]$ with conditions

$$
\begin{aligned}
Y_{j}(a) & =Z_{j}^{1}, & & j=1, \ldots, n-\ell, \\
\Lambda_{i}[Y] & =c_{i}, & i & =1, \ldots, \ell .
\end{aligned}
$$

The restriction to $[0, a]$ of the solution $\tilde{Y}$ to (4.8) solves also the differential system with conditions (4.12), and is its unique solution. Consider now $D Y(t)+$ $A(t) Y(t)=\dot{B}(t)$ on $[b, 1]$, with

$$
\begin{aligned}
Y_{j}(b) & =Z_{j+q}^{1}, & & j=n-\ell-q+1, \ldots, n, \\
\Lambda_{i}[Y] & =c_{i}, & i & =\ell+q+1, \ldots, \ell+q+p,
\end{aligned}
$$

and the $n-\ell-q-p$ equations on $[b, 1]$ that result from

$$
\Lambda_{i}[Y]=c_{i}, \quad i=\ell+q+p+1, \ldots, n,
$$

$$
Y_{n}(t)=\tilde{Y}_{n}(t) \quad \forall t \in \bigcup_{i=\ell+q+p+1}^{n}\left(\operatorname{supp} \Lambda_{i} \cap[0, a]\right) .
$$

Again, $\widetilde{Y}$ restricted to $[b, 1]$ is its unique solution with conditions (4.13) and (4.14). The values $\tilde{Y}_{n}(t)$ appearing here are found in (4.13) as a function of $Z_{j+q}^{1}$, $j=n-\ell-q+1, \ldots, n$, and the Wiener process on $[0, a]$. Therefore, the whole vector $Z^{2}$ is determined by $Z^{1}$ and the increments of $W$ in $] a, b\left[^{c}\right.$. As before, the function $g_{2}\left(z_{1}, \omega\right)$ so defined has a sense for all $z_{1} \in \mathbb{R}^{n+q}$.

STEP 3. The functions $g_{1}$ and $g_{2}$ found in Step 2 satisfy (H1).

PRoOF OF STEP 3. The solution to a linear differential equation depends linearly on the lateral data $c$ [see (2.11)]. Therefore, for each $\omega$ fixed, system (4.10) is linear and it is enough to check that it has a unique solution for $\xi=\eta=0$.

Now, gathering together the lateral conditions (4.12)-(4.14), we obtain the original lateral conditions, so that system (4.10) is equivalent to (4.8) and therefore the solution exists and is unique.

STEP 4. $\quad g_{1}$ and $g_{2}$ satisfy (H2).

Proof of STEP 4. The boundary value problem that defines $g_{1}$ consists of equation $D Y(t)+A(t) Y(t)=\dot{B}(t)$, together with the conditions (4.11). The resulting vector

$$
\left(Y_{1}(a), \ldots, Y_{n-\ell}(a), Y_{n-\ell-q+1}(b), Y_{n}(b)\right)
$$

is absolutely continuous on $\mathbb{R}^{n+q}$, by Proposition 2.1. The proof for $g_{2}$ is analogous, using Remark 2.2. 
Finally, the random vectors $z_{1}-g_{1}\left(z_{2}, \omega\right)$ and $z_{2}-g_{2}\left(z_{1}, \omega\right)$ are independent and have the form $z_{1}-M^{1} z_{2}+U^{1}(\omega)$ and $z_{2}-M^{2} z_{1}+U^{2}(\omega)$, respectively, for some constant matrices $M^{1}$ and $M^{2}$ and some Gaussian absolutely continuous vectors $U^{1}$ and $U^{2}$. We deduce that the $\mathbb{R}^{2 n}$-valued random vector $\left(z_{1}-\right.$ $\left.g_{1}\left(z_{2}, \omega\right), z_{2}-g_{2}\left(z_{1}, \omega\right)\right)$ has a density which is uniformly bounded in $z_{1}$ and $z_{2}$. It follows at once that the function $\delta$ in $(\mathrm{H} 2)$ is bounded.

STEP 5. $\quad g_{1}$ and $g_{2}$ satisfy (H3). Specifically, $\operatorname{det}\left[\mathrm{I}-\nabla g_{1}\left(z_{2}, \omega\right) \nabla g_{2}\left(z_{1}, \omega\right)\right]$ is a constant different from zero.

PROOF OF STEP 5. $g_{1}$ and $g_{2}$ are affine functions of the first argument, with a nonrandom linear coefficient [see (2.11)]. Therefore, $\nabla g_{1}\left(z_{2}, \omega\right)$ and $\nabla g_{2}\left(z_{1}, \omega\right)$ are constant matrices of dimensions $(n+q) \times(n-q)$ and $(n-q) \times(n+q)$ respectively, which we denote simply $\nabla g_{1}$ and $\nabla g_{2}$. We know that the linear system

$$
\begin{aligned}
& z_{1}=g_{1}\left(z_{2}, \omega\right), \\
& z_{2}=g_{2}\left(z_{1}, \omega\right),
\end{aligned}
$$

admits a unique solution. This is equivalent to say

$$
\operatorname{det}\left[\mathrm{I}-\nabla g_{1} \nabla g_{2}\right]=\operatorname{det}\left(\begin{array}{cc}
\mathrm{I} & -\nabla g_{1} \\
-\nabla g_{2} & \mathrm{I}
\end{array}\right) \neq 0
$$

STEP 6. Relation (4.2) holds true.

ProOF OF STEP 6. We can apply Lemma 3.4 and the factorization in (ii) trivially holds. We deduce the relation (4.9) and, by Step 1, that the process $Y$ satisfies the desired property, for $(a, b)$ regular.

Let us now extend Proposition 4.7 to singular pairs $(a, b)$, using an approximation argument. We denote by $\operatorname{Eq}(A, \Lambda)$ our functional boundary value problem relative to the matrix function $A$ and the boundary operator $\Lambda$. The boundary data $c$ will be fixed throughout. Let us call $\Lambda_{1}, \Lambda_{2}, \Lambda_{3}$ the operators associated to the lateral conditions given by (4.5)-(4.7), respectively.

\section{Proposition 4.8. Proposition 4.7 holds also for singular pairs $(a, b)$.}

ProOF. Our initial hypothesis (H0) states that the original problem has one and only one solution, that is, $A(t) \in V_{\Lambda}$, where $V_{\Lambda}$ stands for the set $V$ defined in Section 2.3 , relative to the boundary operator $\Lambda$.

Fix $0 \leq a \leq b$. If the pair $(a, b)$ is singular, then at least one of the problems $\operatorname{Eq}\left(A, \Lambda_{1}\right), \operatorname{Eq}\left(A, \Lambda_{2}\right)$, or $\operatorname{Eq}\left(A, \Lambda_{3}\right)$ is not well posed. We know from Lemma 2.3 
that $V_{\Lambda}$ is open and dense in the space $\mathcal{C}$ of matrices of the form (2.5), for any $\Lambda$. Therefore the set $V:=V_{\Lambda} \cap V_{\Lambda_{1}} \cap V_{\Lambda_{2}} \cap V_{\Lambda_{3}}$ is also open and dense in $\mathcal{C}$.

Let $\left\{A^{N}(t), N \in \mathbb{N}\right\}$ be a sequence of elements of $V$ converging to $A(t) \in \mathcal{C}$. From Proposition 2.4, the corresponding solutions $Y^{N}(t)$ converge to $Y(t)$ in $L^{p}$.

Fix $s \in[a, b]^{c}$ and $r_{1}, \ldots, r_{k} \in(a, b)$. Consider the space $M$ defined in (2.15), based on the coordinates $\left\{s, r_{1}, \ldots, r_{k}\right\} \cup \operatorname{supp} \Lambda$. Let $M^{\prime}$ be the projection of $M$ onto the coordinates $r_{1}, \ldots, r_{k}$. Assume that $Y^{N}(s)$ are nondegenerate [if they are, $Y(s)$ will also be a constant, and there is nothing to prove]. Using Proposition 2.1, the vector $U^{N}:=\left(Y^{N}(s), Y^{N}\left(r_{1}\right), \ldots, Y^{N}\left(r_{k}\right)\right)$ is a Gaussian vector with some density $f^{N}$ with respect to the Hausdorff measure on $\mathbb{R} \times M^{\prime}$. Then there clearly exists an equivalent Gaussian probability with density $f_{0}$ on $\mathbb{R} \times M^{\prime}$ whose first coordinate is not correlated with the remaining ones. Define the probability $Q^{N}$ on $\Omega$ by $d P=\ell^{N} d Q^{N}$, with $\ell^{N}=\frac{f^{N}}{f_{0}}\left(U^{N}\right)$. Then hypothesis 1 of Lemma 3.5 is clearly satisfied with $U_{1}^{N}=Y^{N}(s), U_{2}^{N}=\left(Y^{N}\left(r_{1}\right), \ldots, Y^{N}\left(r_{k}\right)\right)$. Analogously, one can define a probability $Q$ by $d P=\ell d Q$, where $\ell=\frac{f}{f_{0}}(U)$ and $f$ is the density of $U:=\left(Y(s), Y\left(r_{1}\right), \ldots, Y\left(r_{k}\right)\right)$, satisfying hypothesis 2 of Lemma 3.5 with $U_{1}=Y(s)$ and $U_{2}=\left(Y\left(r_{1}\right), \ldots, Y\left(r_{k}\right)\right)$.

We prove now that Hypothesis 3 also holds true: we want to see that if $h$ is a bounded and continuous function, then

$$
\lim _{N \rightarrow \infty} \mathrm{E}_{Q^{N}}\left[h\left(U^{N}, \ell^{N}\left(U^{N}\right)\right)\right]=\mathrm{E}_{Q}[h(U, \ell(U))] .
$$

Notice that

$$
\mathrm{E}_{Q^{N}}\left[h\left(U^{N}, \ell^{N}\left(U^{N}\right)\right)\right]=\int_{M} h\left(u, \ell^{N}(u)\right) f_{0}(u) \mathcal{H}(d u),
$$

where $\mathscr{H}$ denotes the Hausdorff measure, and analogously

$$
\mathrm{E}_{Q}[h(U, \ell(U))]=\int_{M} h(u, \ell(u)) f_{0}(u) \mathcal{H}(d u) .
$$

Convergence (4.15) is then easily derived through the dominated convergence theorem. We can therefore apply Proposition 3.7 to obtain that

$$
\begin{gathered}
L^{2}-\lim _{N} \mathrm{E}\left[F\left(Y^{N}(s)\right) \mid Y^{N}(a), Y^{N}\left(r_{1}\right), \ldots, Y^{N}\left(r_{n}\right), Y^{N}(b)\right] \\
=\mathrm{E}\left[F(Y(s)) \mid Y(a), Y\left(r_{1}\right), \ldots, Y\left(r_{n}\right), Y(b)\right] .
\end{gathered}
$$

Analogously, one obtains the limit

$$
L^{2}-\lim _{N} \mathrm{E}\left[F\left(Y^{N}(s)\right) \mid Y^{N}(a), Y^{N}(b)\right]=\mathrm{E}[F(Y(s)) \mid Y(a), Y(b)] .
$$

We conclude that the conditional independence property can be carried to the limit and this completes the proof of the present proposition and consequently of the necessity in Theorem 4.5 .

The "only if" part of Theorem 4.5 is far easier to prove: 
PROpOsition 4.9. If $\Lambda$ does not preserve $(a, b)$, then relation (4.2) is false.

PROOF. Let us assume, to keep notation simple, that there is only one boundary operator $\Lambda_{1}$, which corresponds to a first-order equation (the general case can be stated similarly). Assume that $\Lambda_{1}$ does not preserve $(a, b)$ and that (4.2) holds true. The corresponding boundary condition can thus be written as

$$
\sum_{t_{j} \in[a, b]^{c}} \alpha_{j} Y\left(t_{j}\right)+\sum_{\left.t_{j} \in\right] a, b[} \alpha_{j} Y\left(t_{j}\right)=c,
$$

where none of the summations is void.

Notice first that

$$
\left(\sum_{\left.t_{j} \in\right] a, b[} \alpha_{j} Y\left(t_{j}\right), Y(a), Y(b)\right)
$$

is an absolutely continuous random vector in $\mathbb{R}^{3}$. This follows easily from Proposition 2.1.

Now, for any bounded and measurable function $\Psi: \mathbb{R} \rightarrow \mathbb{R}$, we have, by the conditional independence hypothesis and relation (4.16), that

$$
\begin{aligned}
& \mathrm{E}\left[\Psi\left(\sum_{\left.t_{j} \in\right] a, b[} \alpha_{j} Y\left(t_{j}\right)\right) \mid Y(a), Y(b)\right] \\
& \quad=\mathrm{E}\left[\Psi\left(\sum_{\left.t_{j} \in\right] a, b[} \alpha_{j} Y\left(t_{j}\right)\right) \mid\{Y(t), t \in] a, b\left[{ }^{c}\right\}\right]=\Psi\left(\sum_{\left.t_{j} \in\right] a, b[} \alpha_{j} Y\left(t_{j}\right)\right) .
\end{aligned}
$$

Taking $\Psi=\mathbf{1}_{[-M, M]}$ for some $M>0$, we get that, on $\left\{\left|\sum_{\left.t_{j} \in\right] a, b[} \alpha_{j} Y\left(t_{j}\right)\right| \leq M\right\}$, which is a set of positive probability, $\sum_{\left.t_{j} \in\right] a, b[} \alpha_{j} Y\left(t_{j}\right)$ is a measurable function of $(Y(a), Y(b))$. In particular, this contradicts the absolute continuity of (4.17).

The next theorem generalizes Theorem 4.5 by allowing the existence of nonpreserving boundary operators, at the price of enlarging the conditioning $\sigma$-field. The result can hardly be called a Markovian type property; nevertheless, it seems interesting in itself, and gives rise to the conjecture contained in Remark 4.12 below.

THEOREM 4.10. Suppose the system

$$
\begin{aligned}
& D Y(t)+A(t) Y(t)=\dot{B}(t), \quad t \in[0,1], \\
& \Lambda[Y]=c
\end{aligned}
$$

satisfies (H0), and let $Y=\{Y(t), t \in[0,1]\}$ be its unique solution. Fix $0 \leq$ $a<b \leq 1$ such that $a, b \notin \operatorname{supp} \Lambda$, and let $g$ be the $\sigma$-field generated by $Y(a), Y(b)$ 
and all variables $Y_{n}(t)$, with $t$ in $\left.\operatorname{supp} \Lambda_{i} \cap\right] a, b\left[\right.$ for some $\Lambda_{i}$ not preserving $(a, b)$. Then,

$$
\sigma\{Y(t), t \in[a, b]\} \frac{\Perp}{g} \sigma\{Y(t), t \in] a, b\left[^{c}\right\} .
$$

The same holds true replacing $] a, b\left[b y[a, b]^{c}\right.$ in the definition of $g$.

PROOF. The result can be proved as Theorem 4.5 with some modifications. We will only give a sketch of the necessary changes in the simple case where there is only one nonpreserving boundary condition, which links one only point $t^{*}$ inside $[a, b]$ with one or more points in $[0, a]$. Specifically, fix $a, b$ and assume that $\ell, p$ and $q$ are as in (4.3) and (4.4), the boundary conditions with support in $[0, a] \cup[b, 1]$ carry the labels $i=\ell+q+p+1, \ldots, n-1$, and that $\Lambda_{n}$ is the nonpreserving condition.

In Step 2, define

$$
\begin{aligned}
Z^{1} & :=\left(\widetilde{Y}_{1}(a), \ldots, \tilde{Y}_{n-\ell-1}(a), \tilde{Y}_{n-\ell-q}(b), \ldots, \widetilde{Y}_{n}(b), \tilde{Y}_{n}\left(t^{*}\right)\right) \in \mathbb{R}^{n+q+1}, \\
Z^{2} & :=\left(\widetilde{Y}_{1}(b), \ldots, \widetilde{Y}_{n-\ell-q-1}(b), \widetilde{Y}_{n-\ell}(a), \ldots, \widetilde{Y}_{n}(a)\right) \in \mathbb{R}^{n-q} .
\end{aligned}
$$

Consider the lateral conditions

$$
\begin{aligned}
Y_{j}(b) & =Z_{j}^{2}, & & j=1, \ldots, n-\ell-q-1, \\
Y_{j}(a) & =Z_{j-q}^{2}, & & j=n-\ell, \ldots, n, \\
\Lambda_{i}[Y] & =c_{i}, & i & =\ell+1, \ldots, \ell+q,
\end{aligned}
$$

on $[a, b]$. This system, as before, defines the function $g_{1}$. To define function $g_{2}$, we consider first $D Y(t)+A(t) Y(t)=\dot{B}(t)$ on $[0, a]$ with conditions

$$
\begin{aligned}
Y_{j}(a) & =Z_{j}^{1}, \quad j=1, \ldots, n-\ell-1, \\
\Lambda_{i}[Y] & =c_{i}, \quad i=1, \ldots, \ell, \\
\Lambda_{n}[Y] & =c_{n}, \\
Y_{n}\left(t^{*}\right) & =\widetilde{Y}_{n}\left(t^{*}\right),
\end{aligned}
$$

and secondly, the system on $[b, 1]$, with

$$
\begin{aligned}
Y_{j}(b) & =Z_{j+q}^{1}, & & j=n-\ell-q, \ldots, n, \\
\Lambda_{i}[Y] & =c_{i}, & i & =\ell+q+1, \ldots, \ell+q+p,
\end{aligned}
$$

and the $n-1-\ell-q-p$ equations on $[b, 1]$ that result from

$$
\begin{array}{ll}
\Lambda_{i}[Y]=c_{i}, & i=\ell+q+p+1, \ldots, n-1, \\
Y_{n}(t)=\tilde{Y}_{n}(t) & \forall t \in \bigcup_{i=\ell+q+p+1}^{n-1}\left(\operatorname{supp} \Lambda_{i} \cap[0, a]\right) .
\end{array}
$$


The claims of Step 4 are also easy to verify using Proposition 2.1, taking into account that we assume $\Lambda$ is in basic form, which implies that $\tilde{Y}_{n}\left(t^{*}\right)$ cannot be a constant.

Another application of the ideas in the proof of Theorem 4.5 provides the following "Markov process" property.

THEOREM 4.11. Fix $0 \leq a \leq 1$ such that $a \notin \operatorname{supp} \Lambda$; the process $Y(t)$ satisfies

$$
\sigma\{Y(t), t \in[0, a]\} \underset{\sigma\{Y(a)\}}{\Perp} \sigma\{Y(t), t \in[a, 1]\}
$$

if and only if for all $i$, either $\operatorname{supp} \Lambda_{i} \subset\left[0, a\left[\right.\right.$, or $\left.\left.\operatorname{supp} \Lambda_{i} \subset\right] a, 1\right]$.

PROOF. One can use the same machinery as in the proof of Theorem 4.5. The "only if" part can be proved within the same lines as Proposition 4.9, whereas for the other implication, if $\operatorname{supp} \Lambda_{i} \subset\left[0, a\left[, i=1, \ldots, \ell\right.\right.$, and $\left.\left.\operatorname{supp} \Lambda_{i} \subset\right] a, 1\right]$, $i=\ell+1, \ldots, n$, then one can take

$$
\begin{aligned}
Z^{1} & :=\left(\tilde{Y}_{1}(a), \ldots, \tilde{Y}_{n-\ell}(a)\right) \in \mathbb{R}^{n-\ell}, \\
Z^{2} & :=\left(\tilde{Y}_{n-\ell+1}(a), \ldots, \widetilde{Y}_{n}(a)\right) \in \mathbb{R}^{\ell},
\end{aligned}
$$

and define $g_{1}$ as a function of $Z^{2}$ and the increments of the Wiener process in $[0, a]$, and $g_{2}$ as a function of $Z^{1}$ and the increments of the Wiener process in $[a, 1]$.

REMARK 4.12. Property (4.18) implies, using Lemma 3.3, that

$$
\sigma\{Y(t), t \in[a, b]\} \frac{\Perp}{\mathscr{H}} \sigma\{Y(t), t \in] a, b\left[^{c}\right\},
$$

where $\mathscr{H}=\sigma\{Y(a), Y(b) ; Y(t), t \in \operatorname{supp} \Lambda \cap[a, b]\}$. We conjecture that this property holds true for a linear functional boundary operator $\Lambda$ supported on any subset of $[0,1]$, and that it is false in general if $\mathscr{H}$ is replaced by a smaller $\sigma$-field. We will show a simple example illustrating the conjecture. Unfortunately, the technique we have employed here does not allow us to prove it.

Consider the process $X(t):=-\int_{0}^{1} W(u) d u+W(t)$, solution of the first-order problem

$$
\begin{aligned}
& \dot{X}(t)=\dot{W}(t), \quad t \in[0,1], \\
& \int_{0}^{1} X(u) d u=0,
\end{aligned}
$$

in which the support of the boundary operator is the whole interval $[0,1]$. Fix $a \in] 0,1[$, and set

$$
\mathcal{g}:=\sigma\{X(u), u \in[0, a] ; X(1)\} \quad \text { and } \quad \mathscr{H}:=\sigma\{X(u), u \in[a, 1]\} .
$$


We have trivially $\mathscr{g} \Perp_{\mathscr{H}} \mathscr{H}$, but the conditioning $\mathscr{H}$ cannot be replaced by the smaller $\sigma$-field $\mathscr{H}^{\prime}:=\sigma\{X(u), u \in[a, 1]-[s, t]\}$. Indeed, it is easy to see that

$$
T:=\mathrm{E}\left[\int_{0}^{a} X(u) d u \mid \mathcal{H}\right]=-\int_{a}^{1} X(u) d u,
$$

and one can check that $T$ is not $\mathscr{H}^{\prime}$-measurable: choose $\omega^{1}, \omega^{2} \in C_{0}([0,1] ; \mathbb{R})$ such that $\omega^{1} \equiv \omega^{2}$ on $] s, t\left[{ }^{c}\right.$ and $\omega^{1}<\omega^{2}$ on $] s, t$. An easy computation gives

$$
T\left(\omega^{2}\right)-T\left(\omega^{1}\right)=a \int_{s}^{t}\left(\omega^{2}-\omega^{1}\right)(u) d u>0 .
$$

Now, due to the continuity of $T$ as a functional on $C_{0}([0,1] ; \mathbb{R})$, it is possible to find two open balls, centered at $\omega^{1}$ and $\omega^{2}$, such that their images through $T$ take values in two disjoint intervals of $\mathbb{R}$. We conclude that $\mathcal{G}$ and $\mathscr{H}$ are not conditionally independent given $\mathscr{H}^{\prime}$.

\section{REFERENCES}

[1] Alabert, A. and Ferrante, M. (1998). A conditional independence property for the solution of a linear stochastic differential equation with lateral conditions. In Stochastic Analysis and Related Topics VI (L. Decreusefond, J. Gjerde, B. Øksendal and A. S. Üstünel, eds.) 159-173. Birkhäuser, Boston.

[2] Alabert, A., Ferrante, M. and Nualart, D. (1995). Markov field property of stochastic differential equations. Ann. Probab. 23 1262-1288.

[3] Alabert, A. and Marmolejo, M. A. (1999). Reciprocal property for a class of anticipating stochastic differential equations. Markov Process. Related Fields 5 331-356.

[4] Alabert, A. and Nualart, D. (1997). A second order Stratonovich differential equation with boundary conditions. Stochastic Process. Appl. 68 21-47.

[5] Bernstein, S. (1932). Sur les liaisons entre les grandeurs aléatoires. In Proceedings of the International Congress of Math. 288-309. Zurich.

[6] Chow, S.-N. and LASOTA, A. (1973). On boundary value problems for ordinary differential equations. J. Differential Equations 14 326-337.

[7] Conti, R. (1961). Problèmes linéaires pour les équations différentielles ordinaires. Math. Nachr. 23 161-178.

[8] FEČKAN, M. (1995). On the continuous dependence of solutions of nonlinear equations. J. Math. Anal. Appl. 194 578-596.

[9] Ferrante, M. and Nualart, D. (1997). An example of a non-Markovian stochastic twopoint boundary value problem. Bernoulli 3 371-386.

[10] Goggin, E. (1994). Convergence in distribution of conditional expectations. Ann. Probab. 22 $1097-1114$.

[11] Hönig, C. S. (1973). The Green function of a linear differential equation with a lateral condition. Bull. Amer. Math. Soc. 79 587-593.

[12] JAmison, B. (1970). Reciprocal processes: The stationary Gaussian case. Ann. Math. Statist. 41 1624-1630.

[13] KNUDSEn, T. S. (1998). Convergence in the mean of conditional expectations. Preprint.

[14] Krener, A. J. (1997). Reciprocal diffusions in flat space. Probab. Theory Related Fields 107 243-281. 
[15] Krener, A. J., Frezza, R. and Levy, B. C. (1991). Gaussian reciprocal processes and selfadjoint stochastic differential equations of second order. Stochastics Stochastics Rep. 34 29-56.

[16] Nualart, D. and PARdoux, E. (1991). Boundary value problems for stochastic differential equations. Ann. Probab. 19 1118-1144.

[17] Nualart, D. and Pardoux, E. (1991). Second order stochastic differential equations with Dirichlet boundary conditions. Stochastic Process. Appl. 39 1-24.

[18] OCONE, D. and PARdoux, E. (1989). Linear stochastic differential equations with boundary conditions. Probab. Theory Related Fields 82 489-526.

[19] RUSSEK, A. (1980). Gaussian $n$-Markovian processes and stochastic boundary value problems. Z. Wahrsch. Verw. Gebiete 53 117-122.

[20] Thieullen, M. (1993). Second order stochastic differential equations and non-Gaussian reciprocal diffusions. Probab. Theory Related Fields 97 231-257.

[21] Zambrini, J.-C. (1989). Probability and analysis in quantum physics. In Stochastic Analysis, Path Integration and Dynamics (K. D. Elworthy and J.-C. Zambrini, eds.) 223-242. Longman, Harlow, UK.

DEPARTAMENT DE MATEMÀtiques

UNIVERSITAT AUTÒNOMA DE BARCELONA

08193 BELLATERRA

Catalonia

E-MAIL: alabert@mat.uab.es

WEB: mat.uab.es/alabert
Dipartimento di MATEMATICA PURA ED APPLICATA

UNIVERSITÀ DEGLI STUdi Di PAdOVA

VIA BELZONI 7

35131 PADOVA

ITALY

E-MAIL: ferrante@math.unipd.it

WEB: math.unipd.it/ferrante/index.html 